\title{
The local treatment of DCIS should be different than that of early-stage invasive cancer
}

\author{
Janusz Piekarski
}

Surgical methods currently applied in the local treatment of pre-invasive ductal carcinoma are largely the same as those used in early-stage invasive breast cancer. Both conditions are treated either with tumor excision (combined with radiation therapy) or with mastectomy. Comparable proportions of DCIS and early-stage breast cancer patients receive the most aggressive form of treatment. Nearly $30 \%$ of the former undergo mastectomy. A question thus presents itself: is this the way it should be?

The purpose of DCIS treatment is to prevent its progression to invasive disease or detect a hidden invasive component. However, in and of itself, DCIS is not a terminal condition and does not directly put life at risk. Indeed, it may be a precursor of invasive cancer, but progression is known to occur in as few as $20-30 \%$ of untreated patients. At the same time, it is a quite heterogeneous diagnostic category. Some ductal carcinomas in situ with the lowest degree of malignancy behave more like atypias and show a similar risk of progression, only slightly higher than that for an average 65-year-old woman in the general population. It has even been argued that these DCIS cases are not properly carcinomas at all; labeling them as "cancer" provokes disproportionate fear in patients and compels physicians to take unnecessary and excessive treatment measures. In order to change that, it is first crucial to modify the terminology we use: changes with a very low risk of progression toward invasive carcinoma should be labeled as indolent lesions of epithelial origin (IDLE). To conclude, the local treatment of pre-invasive carcinoma in most cases should differ from that of early-stage invasive cancer. Aggressive methods should be avoided unless specifically required.

NOWOTWORY J Oncol 2017; 67, 1:71-73

Key words: ductal carcinoma, cancer in situ, treatment, indolent lesion of epithelial origin (IDLE)

When we ask ourselves whether the local treatment of pre-invasive ductal carcinoma should be the same as that of early-stage invasive cancer, the answer that immediately comes to mind is a resounding "no". Why? It is enough to take a look at available diagnostic and treatment guidelines, which all discuss principles applicable to DCIS and invasive cancer under separate sections. Examples there are aplenty, including the guidelines issued by the Polish Oncology Union [1] and the National Consultant for Cancer Surgery, American recommendations from the National Comprehensive Cancer Network [2] and the MD Anderson Cancer Center [3], as well as German guidelines from the Arbeitsgemeinschaft Gynäkologische Onkologie [4]. The
Polish Oncology Union recommends that the choice of treatment should rely on the Van Nuys prognostic indicator (VNPI), which takes into account tumor size, the width of clear surgical margins, nuclear grade classification, and patient age [1]. American and German guidelines do not use the VNPI, but also suggest that physicians should base their treatment decisions on tumor size and surgical margins.

However, a quick look at the surgical methods currently employed in DCIS treatment reveals that they are largely the same as those used in patients with early-stage invasive cancer. These include mastectomy or tumor excision with (or, in exceptional cases, without) accompanying radiation therapy. Interestingly, aggressive treatments are used with similar fre- 
quency in patients with DCIS and early-stage invasive cancer. A thought-provoking study on the subject was published in the Journal of the National Cancer Institute in September 2015 [5], where Worni et al. tallied up SEER database records on DCIS treatment for the period between 1991-2010. The study identified a total of 121,080 patients; $23.8 \%$ underwent unilateral mastectomy, $4.5 \%$ had bilateral mastectomy, $43 \%$ were treated with tumor excision and radiation therapy, and excision alone was performed in $26.5 \%$. $2.3 \%$ were not operated on at all. These averaged data, however, fail to reflect the changes in treatment methods that occurred over the analyzed period. Closer inspection reveals that the proportion of patients treated with tumor excision and radiation therapy doubled, rising from $24.2 \%$ to $46.8 \%$ over the time span in question. The number of amputations, on the other hand, halved, dropping from $44.9 \%$ to a mere $19.3 \%$. Interestingly, however, the percentage of bilateral mastectomies due to non-invasive ductal carcinoma grew from $0 \%$ to $8.5 \%$. These figures can be summed up very briefly. DCIS patients are being treated with very aggressive methods; nearly $30 \%$ have (unilateral or bilateral) mastectomies and 50\% undergo tumor excision followed by radiation therapy [5]. We might thus conclude that, in actual fact, the topical treatment of DCIS today is exactly the same as that of early-stage invasive cancer. Is this the way it should be? In order to find an answer, we should first consider the goal of treatment in both conditions.

The purpose of therapy in invasive breast cancer is clear. We are dealing with a potentially fatal disease that, in 2012 alone, caused as many as 915 thousand deaths throughout the European Union [6]. Treatment is geared toward preventing death and prolonging life expectancy; aggressive measures are thus fully justified. This is why the comparison of methods used in the treatment of early-stage cancer in 13 European Breast Unit centers does not come as a surprise. More than $25 \%$ of patients are reported to undergo mastectomy [7]. The figure is well warranted; what might raise a few eyebrows, however, is the fact that it is lower than the percentage of mastectomies among DCIS patients in the US [5].

What is the objective of treatment in DCIS? The goal is to prevent progression to invasive disease or detect a hidden invasive component [2]. In and of itself, DCIS is not a fatal condition and does not pose a risk to patients'lives. Diagnosis and prevention are important objectives, of course, but do they really warrant a $30 \%$ amputation rate, including bilateral mastectomy in as many as $10 \%$ of patients $[5,8]$ ? Mortality among women treated for DCIS reaches $3.3 \%$ after 20 years [9]. Importantly, treatment methods do not seem to have a statistically significant impact on treatment results. The latter are normally very good. But why shouldn't they, considering that the disease is not life-threatening to begin with? What does pose a threat to patients' lives are possible diagnostic errors (a failure to detect invasion and take appropriate action) and relapse in the form of invasive disease.
Indeed, DCIS is recognized as a precursor of cancer, but progression only occurs in as few as $20-30 \%$ of untreated patients [5]. What are the actual figures? As reported by Worni et al. [5], approximately 50-60 thousand new DCIS cases are diagnosed in the US every year. In the absence of treatment, progression may thus be expected to occur in about 15 thousand. This means that as many as 45 thousand patients every year are subjected to aggressive and often mutilating treatments for a disease that does not even threaten their lives.

Of course, a small risk of progression does exist and one may well argue that all measures should be taken to make sure it does not materialize. It is worth asking, however, whether we treat every high-risk patient in the same way. Do we always resort to equally aggressive treatment methods? Women with $B R C A 1$ and $B R C A 2$ gene mutations are particularly likely to develop breast cancer; more than $80 \%$ are at risk of the disease. It turns out, however, that only $20 \%$ undergo prophylactic bilateral mastectomy [10]. How does this compare to the situation of DCIS patients, where the likelihood of progression stands at c. $20-30 \%$ and bilateral and unilateral mastectomy is performed, respectively, in 10 and $20 \%$ of cases? For women with atypical hyperplasia, 35\% of whom will develop breast cancer within the next 30 years [11], all we recommend is regular observation! It is difficult to resist the impression that we may be guilty of unequal treatment and inconsistency in our handling of high-risk patients.

The question of whether we might be treating DCIS patients too aggressively is asked with increasing frequency. It has been the subject of studies published in top medical journals, such as the New England Journal of Medicine [12], Lancet Oncology [13], and the Journal of the National Cancer Institute [14]. Scholars point out that, paradoxically, the problem might lie in the efficiency, or rather, the hyper-efficiency of our screening systems. Cancer screening relies on several premises. The fundamental one is that progression toward malignant disease is an ordered and gradual process. The second assumes that screening allows identification of cancer at an early stage. The last states that early detection and effective treatment lead to a significant decrease in mortality rates [13]. The three premises have been confirmed in clinical practice for cervical and colorectal cancer. Early detection of pre-malignant conditions has led to reduction of the incidence of related cancer cases and resulted in decreased mortality rates. In breast cancer, however, the impact of screening on mortality is much less significant than expected. Importantly, a major increase in the detection of early-stage (invasive and non-invasive) cases has failed to translate into a reduced number of advanced cases and generalized cancer incidence [15]. It is very likely that our screening tests are too sensitive and detect many early conditions that would never pose any threat to patients' lives. Once detected, they are treated with effective but aggressive methods that considerably affect patient health. 
This approach to diagnosis and treatment may bring a certain sense of satisfaction. We have been very successful after all. Breast cancer mortality rates are going down. We should be aware, however, that the phenomenon might stem not from the lower overall number of deaths, but the increased detection of early cases that are not life-threatening to begin with. An old joke tells of a suit vendor who decided to increase the discounts on sale items. A suit cost 100 PLN before and 80 PLN after price reduction. In order to further increase the discount, the final price was left unchanged ( 80 PLN), but the original price on the label increased to 120 PLN. Isn't our success in the treatment of breast cancer somewhat similar?

It seems that DCIS is a very heterogeneous diagnostic category. Some ductal carcinomas in situ with the lowest degree of malignancy behave more like atypias and show a similar risk of progression, only slightly higher than that for an average 65-year-old woman in the general population [13]. It has been argued that these DCIS cases are not properly carcinomas at all; labeling them as "cancer" provokes disproportionate fear in patients and compels physicians to take unnecessary and excessive treatment measures $[12,13]$. Instead of aggressive oncological treatment, DCIS patients should be recommended to lead an active lifestyle, reduce their BMI to max. 25, and take hormonal medication to lower the risk of progression to invasive disease [12]. The effectiveness of tamoxifen in the prevention of breast cancer is the same as that of radiation therapy in preventing relapse after tumor excision. Surely, there is no need for mastectomy, and yet it is still performed in $30 \%$ of DCIS patients.

To recapitulate: efficient imaging techniques have increased the detection of DCIS; in the absence of treatment, only $1 / 4$ of DCIS cases progress to invasive cancer; current treatment is effective, but very aggressive, and, in most cases, probably unwarranted.

In order to change that, it is first crucial to modify the terminology we use. The "CANCER" label wreaks havoc and is not always clinically justified. Changes with a very low risk of progression toward invasive carcinoma should be labeled as indolent lesions of epithelial origin (IDLE) instead [13].

Should the local treatment of DCIS be the same as that of early-stage invasive cancer? In most cases, NO. Aggressive methods should be avoided unless specifically required.

\section{Conflict of interest: none declared}

\section{Prof. Janusz Piekarski, MD, PhD}

Department of Surgical Oncology, Medical University of tódź

Paderewskiego 4, 93-509 Łódź, Poland

e-mail:januszpiekar@poczta.onet.pl

Received \& Accepted: 5 Jul 2016

Based on the presentation at the IV Annual Conference of the Nowotwory Journal of Oncology, 'Oncological Debates', held in Warszawa, 8-9th April 2016

\section{References}

1. Jassem J, Krzakowski M, Bobek-Billewicz B et al. Rak piersi. In: Krzakowski M, Warzocha K (red.) Zalecenia postępowania diagnostyczno-terapeutycznego w nowotworach złośliwych 2013 rok. Gdańsk: Via Medica; 2013: 213-263.

2. National Comprehensive Cancer Network. NCCN guidlines: breast cancer Version I.2016 (www.nccn.org).

3. MD Anderson Cancer Center. Cancer treatment algorithms: breast cancer - noninvasive, version 3/31/2015. www.mdanderson.org.

4. Diagnosis and treatement of patients with primary and metastatic breast cancer. Vs 1.2016, https://issuu.com/agomamma.

5. Worni M, Akushevich I, Greenup R et al. Trends in treatment patterns and outcomes for ductal carcinoma in situ. J Natl Cancer Inst 2015; 107: djv263.

6. Schuz J, Espina C, Villain R et al. European Code against Cancer 4th edition: 12 ways to reduce your cancer risk. Cancer Epidemiol 2015; 39 Suppl 1: S1-10.

7. Garcia-Etienne CA, Tomatis M, Heil J et al. Mastectomy trends for early-stage breast cancer: a report from the EUSOMA multi-institutional European database. Eur J Cancer 2012; 48: 1947-1956.

8. Rutter CE, Park HS, Killelea BK et al. Growing use of mastectomy for ductal carcinoma in-situ of the breast among young women in the United States. Ann Surg Oncol 2015; 22: 2378-2386.

9. Narod SA, lqbal J, Giannakeas $V$ et al. Breast cancer mortality after a diagnosis of ductal carcinoma in situ. JAMA Oncol 2015; 1: 888-896.

10. Friebel TM, Domchek SM, Neuhausen SL et al. Bilateral prophylactic oophorectomy and bilateral prohpylactic mastectomy in a prospective cohort of unaffected BRCA1 and BRCA2 mutation carriers. Clin Breast Cancer 2007; 7: 875-882.

11. Degnim AC, Visscher DW, Berman HK et al. Stratification of breast cancer risk in women with atypia: a Mayo cohort study. J Clin Oncol 2007; 25: 2671-2677.

12. Merrill AL, Esserman L, Morrow M. Clinical decissions. Ductal carcinoma in situ. N Engl J Med 2016; 374: 390-392.

13. Esserman LJ, Thompson IM, Reid B et al. Addressing overdiagnosis and overtreatment in cancer: a prescription for change. Lancet Oncol 2014; 15: e234-242.

14. Morrow M, Katz SJ. Addressing overtreatment in DCIS: what should physicians do now? J Nat/ Cancer Inst 2015; 107: djv290.

15. Ozanne EM, Shieh Y, Barnes J et al. Characterizing the impact of 25 years of DCIS treatment. Breast Cancer Res Treat 2011; 129: 165-173.

16. Vogel VG, Constantino JP, Wickerham DL et al. Effects of tamoxifen vs raloxifene on the risk of developing invasive breast cancer and other disease outcomes: the NSABP Study of Tamoxifen vs Raloxifene (STAR) P-2 trial. JAMA 2006; 295: 2727-2741. 